\title{
Inertial effects on thermal transport in superhydrophobic microchannels
}

\author{
Adam Cowley, Daniel Maynes*, Julie Crockett \\ Department of Mechanical Engineering \\ Brigham Young University \\ Provo, UT 84602
}

\begin{abstract}
This paper presents a numerical investigation on the effects of inertia for laminar fully developed flow in superhydrophobic microchannels where the surface structure consists of a square array of square pillars aligned with the flow direction. Infinite parallel plate channel flow is considered where the flow is assumed to be non-wetting and the meniscus is idealized as flat. A constant heat flux condition is imposed at the tops of the posts whereas the air/liquid interface is assumed to be adiabatic. A wide range of Peclet numbers (1 - 10000), relative channel spacing sizes (3 orders of magnitude), and solid fractions $(0.02-0.9)$ are considered. The effect of these on friction factor-Reynolds number product, Nusselt number, hydrodynamic slip length and temperature jump length, is explored. Frictional drag and convective heat transfer are reduced for all cases explored. These reductions are greater for small solid fractions, small relative channel sizes, and low Peclet numbers. Interestingly, over a wide range of explored parameters the results correspond well with the analytical diffusion dominated results present in the literature. It is only at the highest Peclet numbers and smallest relative channel sizes that the results deviate significantly from the diffusion dominated Stokes flow scenario. A mapping is presented that illustrates for which parameter ranges the influence of inertia and relative channel size become significant.
\end{abstract}

Keywords: superhydrophobic, hydrodynamic slip length, temperature jump length, convection, heat transfer, microchannel

\section{Nomenclature}

A Slope for hydrodynamic slip length linear regression $b$ Hydrodynamic slip length

$b_{t}$ Temperature jump length

$B$ Intercept for hydrodynamic slip length linear regression

$c_{p}$ Fluid specific heat

$C$ Slope for temperature jump length linear regression

$D$ Intercept for temperature jump length linear regression

$D_{h}$ Channel hydraulic diameter $(2 H)$

$f$ Darcy friction factor

$F_{s}$ Solid fraction $\left(s^{2} / L^{2}\right)$

$\hat{F}_{s, f R e} 5 \%$ Stokes flow cuttoff solid fraction for $f R e$

$\hat{F}_{s, N u} 5 \%$ Stokes flow cuttoff solid fraction for $\overline{N u}$

$h$ Convection coefficient

$\bar{h}$ Average convection coefficient

$H$ Channel height

$k_{f}$ Fluid thermal conductivity

$L$ Module length (pitch)

$\dot{m}$ Mass flow rate in computational domain

$N u$ Nusselt number $\left(h D_{h} / k_{f}\right)$

$\overline{N u}$ Average Nusselt number $\left(\bar{h} D_{h} / k_{f}\right)$

$p$ Pressure

$\hat{p}$ Periodic pressure

*Corresponding Author. Tel: +1 8014223843

Email address: maynes@byu.edu (Daniel Maynes)
Pe Peclet number $(R e P r)$

$\operatorname{Pr}$ Prandtl number $(\nu / \alpha)$

$q_{c}^{\prime \prime}$ Heat flux through composite interface

$q_{s}^{\prime \prime}$ Heat flux through solid/liquid interface

$Q$ Overall rate of heat addition to control volume

Re Reynolds number $\left(\rho \bar{u} D_{h} / \mu\right)$

$s$ Post top side length

$T$ Fluid temperature

$\hat{T}$ Periodic fluid temperature

$T^{*}$ Average fluid temperature profile

$\bar{T}_{c}$ Average composite interface temperature

$\bar{T}_{s}$ Average solid/liquid interface temperature

$u$ Streamwise fluid velocity

$u^{*}$ Average fluid velocity profile

$u_{s}$ Apparent slip velocity

$\bar{u}$ Mean fluid velocity

$U_{s}$ Normalized slip velocity $\left(u_{s} / \bar{u}\right)$

$v$ Interface normal fluid velocity

$\vec{V}$ Fluid velocity vector

$w$ Transverse fluid velocity

$W_{m}$ Relative module length $\left(L / D_{h}\right)$

$x$ Streamwise coordinate

$y$ Interface normal coordinate

$z$ Transverse coordinate

Greek Symbols

$\alpha$ Thermal diffusivity $\left(k_{f} / \rho c_{p}\right)$ 
$\beta$ Pressure drop per module length

$\gamma$ Temperature shift per module length

$\mu$ Dynamic fluid viscosity

$\nu$ Kinematic fluid viscosity $(\mu / \rho)$

$\rho$ Fluid density

\section{Introduction}

Superhydrophobic (SH) surfaces have been of interest recently due to their unique water repelling capabilities. SH surfaces hold promise for a wide range of applications including: self cleaning surfaces, water repellency, drag reduction, anti-icing, condensation enhancement, and labon-chip devices. SH surfaces function through a combination of micro/nano-scale texturing and a hydrophobic coating. When water comes in contact with a $\mathrm{SH}$ surface, the water rests on top of the texturing and (due to surface tension) does not penetrate into the cavities between the textured features. Thus, the cavities remain filled with gas (usually air) and a meniscus is formed between the water and the gas. When this behavior occurs, the surface is said to be in the Cassie-Baxter state [1]. The surface is further deemed superhydrophobic when the contact angle between it and a sessile droplet of water is greater than nominally $145^{\circ}[2]$.

When a liquid flows over a $\mathrm{SH}$ surface and the CassieBaxter state is maintained, the hydrodynamics of the flow can be dramatically altered. Much of the working liquid is in contact with the gas in the cavities, which has a viscosity much less than that of the liquid, and therefore a nearly shear free condition exists at the gas/liquid interfaces. This can result in an overall frictional drag reduction for the system and from a macroscopic view there exists an average non-zero slip velocity $\left(u_{s}\right)$ at the surface. These unique hydrodynamics have been extensively studied for laminar flow over SH surfaces [3-12]. Additionally, much work has focused on drag reduction using $\mathrm{SH}$ surfaces in the turbulent flow regime [13-16]. Substantial work has also been performed regarding drops [17-21] as well as jet impingement on SH surfaces [22-25].

Multiple studies have focused on determining the hydrodynamic slip length for flow over surfaces with mixed boundary conditions $[5,8,9,11,26-29]$. The slip velocity can be expressed as

$$
u_{s}=\left.b \frac{d u^{*}}{d y}\right|_{y=0}
$$

where $u^{*}$ is the average velocity profile, $y$ is the coordinate normal to the composite boundary of the surface, and $b$ is the hydrodynamic slip length. The slip length (originally presented by Navier [30]) is the distance below the surface where the no-slip condition would be regained if the average velocity profile at the composite interface were extrapolated (see Fig. 1 left panel). This quantity is a common metric used to define the frictional drag reduction achieved by a $\mathrm{SH}$ surface on a macroscopic scale, where a larger slip length corresponds to a larger drag reduction. It has been found that the geometry and orientation of the surfaces structures, flow Reynolds number, meniscus shape, and channel size (for internal flow) all affect the hydrodynamic slip length [7-10, 28, 29].

These surfaces can also have a significant effect on heat transfer. If the $\mathrm{SH}$ surface substrate is metal and the hydrophobic coating is thin, then the effective thermal conductivity of the solid features is orders of magnitude greater than that of the gas in the cavities. The cavities act as a barrier to heat transfer and increase the overall thermal resistance through the surface. An experimental study found the evaporation times of sessile droplets on $\mathrm{SH}$ surfaces to be substantially greater than those on smooth surfaces [31], while others have observed that SH surfaces change frost formation behavior $[32,33]$.

Various studies seek the apparent temperature jump length (or thermal slip length) to describe the macroscopic effect of SH surfaces on thermal transport [34-38]. A larger temperature jump length corresponds to a greater overall convective heat transfer reduction. Temperature jump length is analogous to the concept of hydrodynamic slip length and is defined in the following equation [36]

$$
\bar{T}_{s}-\bar{T}_{c}=-\left.b_{t} \frac{d T^{*}}{d y}\right|_{y=0}
$$

where $\bar{T}_{s}$ is the average temperature of the solid/liquid interface, $\bar{T}_{c}$ is the average temperature of the solid/liquid and gas/liquid (or composite) interface, $T^{*}$ is the average temperature profile, and $b_{t}$ is the temperature jump length (see Fig. 1 right panel).

Enright et al. [36] provide analytical and/or approximate expressions for the hydrodynamic slip length and the temperature jump length for both constant heat flux and constant temperature conditions for posts and ribs. The analysis assumes a diffusion dominated scenario and a semi-infinite domain. In general, both slip lengths increase with decreasing solid fraction. Ng and Wang [37] semi-analytically find the temperature jump length for a constant temperature boundary condition on square and circular posts and holes using a diffusion dominated solution as well. The effects of gas cavities with finite thermal conductivity [37] and evaporation at the menisci [38] have been explored for rib/cavity surfaces and both effects tend to reduce the effective temperature jump length. A few studies have also investigated the thermal transport for scenarios with imposed hydrodynamic and thermal slip lengths [39-42].

Since SH surfaces can potentially reduce drag, they present a viable option for passively reducing the pressure drop for driving fluid in channels. This is particularly attractive in microfluidic devices where the necessary driving pressure can become very large. Thermal management is of high importance in many microfluidic devices and the convective heat transfer over SH surfaces must be understood in order to appropriately design such devices. 

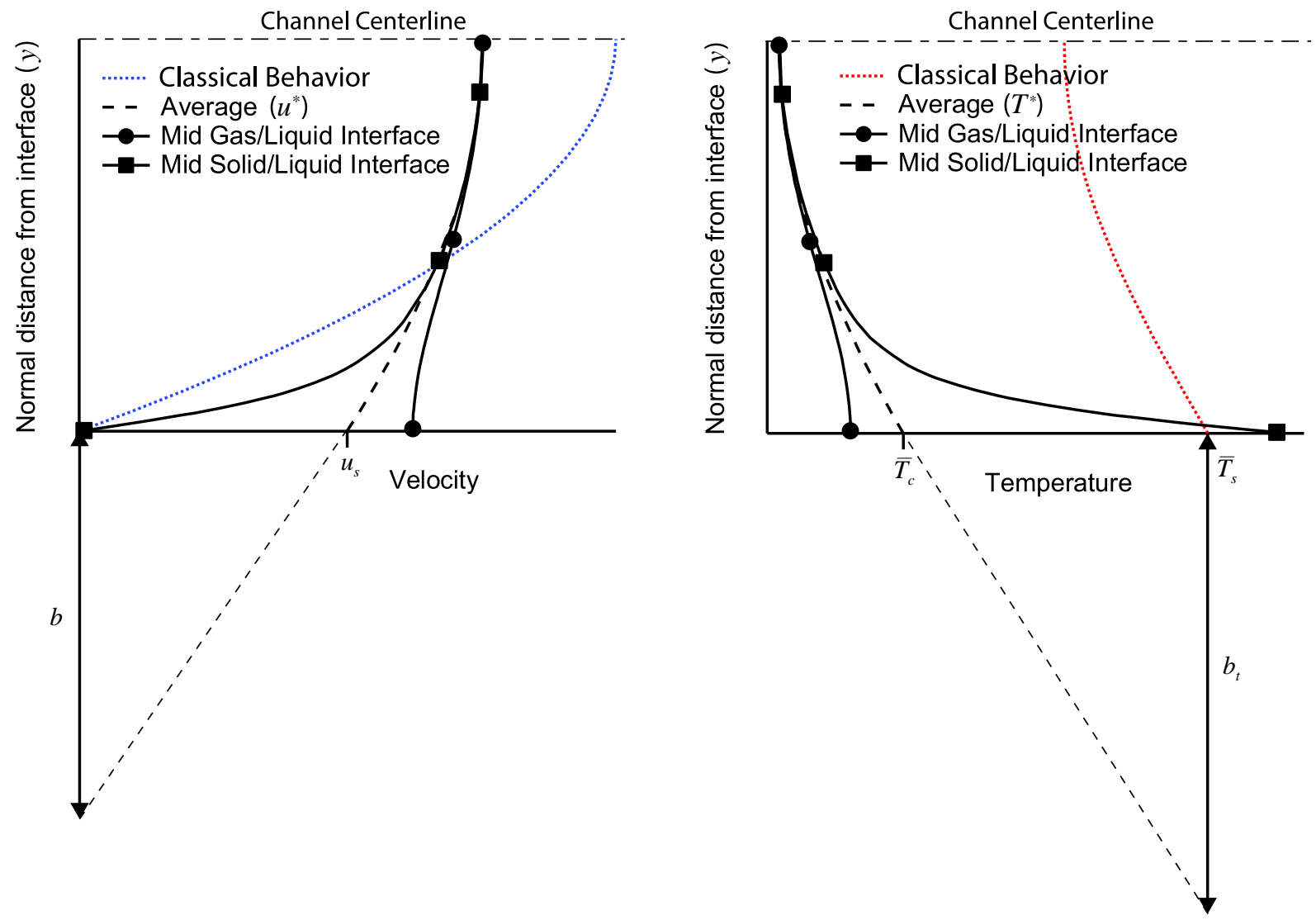

Figure 1: Graphical description of the hydrodynamic slip length (left panel) and temperature jump length or thermal slip length (right panel) for laminar internal flow and a constant heat flux thermal boundary condition. 
Moreira and Bandaru [43] utilize an effective medium approach to account for the differing thermal conductivities of the gas and solid regions as well as substrate size for transverse ribbed SH walls in two dimensional channel flow. They find that the decreased effective thermal conductivity of the substrate plays a larger role than the modified hydrodynamics on the overall thermal transport. A singular experimental study for laminar channel flow by Rosengarten et al. [44] examines heat transfer in a PDMS $\mathrm{SH}$ microchannel. They find the heat transfer to be reduced for the SH microchannel.

Several additional studies have investigated the convective heat transfer in SH microchannels [34, 35, 45-47]. Along with the SH surface structure and thermal boundary condition, several non-dimensional parameters are important in characterizing the thermal transport in $\mathrm{SH}$ microchannels. These include: the solid fraction $\left(F_{s}\right)$, which is the ratio of the solid/liquid interface area to the total composite interface area; the relative module length to channel size ratio $\left(W_{m}=L / D_{h}\right)$, where $L$ is a single module length and $D_{h}$ is the channel hydraulic diameter; and Peclet number $\left(P e=R e P r=\rho \bar{u} D_{h} / \alpha\right)$, where $R e$ is Reynolds number based on the hydraulic diameter, $\mathrm{Pr}$ is Prandtl number, $\rho$ is the fluid density, $\bar{u}$ is the mean channel velocity, and $\alpha$ is the fluid thermal diffusivity.

Maynes et al. [45] numerically investigated flow over ribs aligned transverse to the flow direction where the ribs were maintained at a constant temperature boundary condition. Maynes and Crockett [34] analytically investigated the constant heat flux boundary condition for flow over ribs oriented parallel to the flow direction. Maynes et al. [46] explored the constant heat flux scenario analytically for flow over ribs oriented transverse to the flow direction, however, axial fluid conduction was neglected. Cowley et al. [35] extended the work of Maynes et al. [46] for the fully developed region by including axial fluid conduction computationally. Cheng et al. [47] numerically explored the hydrodynamics and heat transfer for square posts and holes as well as both longitudinal and transverse ribs where a constant temperature thermal boundary condition was considered. However, they only look at one relative module length.

These studies $[34,35,45-47]$ all find that Nusselt number $\left(N u=h D_{h} / k_{f}\right)$ is locally enhanced over the solid/liquid interface, where $h$ is the convection coefficient and $k_{f}$ is the fluid thermal conductivity. However, the average Nusselt number $(\overline{N u})$ is less than that of a classical smooth wall channel when averaged over the entire composite interface.

While the convective heat transfer (or average Nusselt number) is reduced for $\mathrm{SH}$ microchannels, the drag reduction may be sufficient enough that the increase in mass flow rate for a similar driving pressure (or other driving force) can result in an overall heat transfer enhancement. Lam et al. show that such an enhancement can be achieved for microchannels where Galinstan (a liquid metal) is the working fluid [48].

The goal of this paper is to extend the literature for convective heat transfer in SH microchannels by investigating the influence of inertia (high Peclet number flow) for a square array of square posts maintained at a constant heat flux boundary condition as well as to explore how inertial influences change with varying relative module length. We quantify this by determining the corresponding average Nusselt number and temperature jump length for each scenario considered. Over $200 \mathrm{Pe}, F_{s}$, and $W_{m}$ combinations are explored, encompassing the range of realizable parameters for laminar flow. Such a study has not yet been done for this surface structure/thermal boundary condition combination, nor one that is so exhaustive. This study compares results to the diffusion dominated work of Enright et al. [36] and illustrates for which parameter ranges the influence of inertia and relative channel size become significant.

\section{Methodology}

In this paper a steady, pressure driven, laminar flow through infinite parallel plates of wall-to-wall spacing $H$ is analyzed. Fluid properties are considered constant and no volumetric heat sources are present. Thermocapillary stresses are not considered. The flow is idealized to remain in the Cassie-Baxter state and have a flat meniscus at the gas/liquid interface. The meniscus is assumed to be shear-free and adiabatic as well. The surface structure examined consists of a square array of square posts and the flow is aligned with the square array (see Figure 2). The Navier-Stokes, continuity and energy equations govern the hydrodynamics and heat transfer for the given scenario

$$
\begin{gathered}
\rho(\vec{V} \cdot \nabla \vec{V})=-\nabla p+\mu \nabla^{2} \vec{V} \\
\nabla \cdot \vec{V}=0 \\
\rho c_{p}(\vec{V} \cdot \nabla T)=k_{f} \nabla^{2} T
\end{gathered}
$$

where $\rho$ is the fluid density, $p$ is pressure, $\mu$ is fluid viscosity, $c_{p}$ is the fluid specific heat, $k_{f}$ is the thermal conductivity of the fluid and $T$ is temperature. $\vec{V}$ is the velocity vector field comprised of the streamwise, interface-normal and spanwise scalar components of velocity $(u, v$, and $w)$ respectively.

The periodic control volume used for the numerical study is depicted in Figure 3. As described by Patankar et al. the velocity field will repeat itself at each module length $(L)$ [49] and while the pressure field itself is not periodic, the pressure drop across each module is constant.

$$
\begin{aligned}
& \frac{p(x, y, z)-p(x+L, y, z)}{L}= \\
& \quad \frac{p(x+L, y, z)-p(x+2 L, y, z)}{L}=\ldots=\beta
\end{aligned}
$$




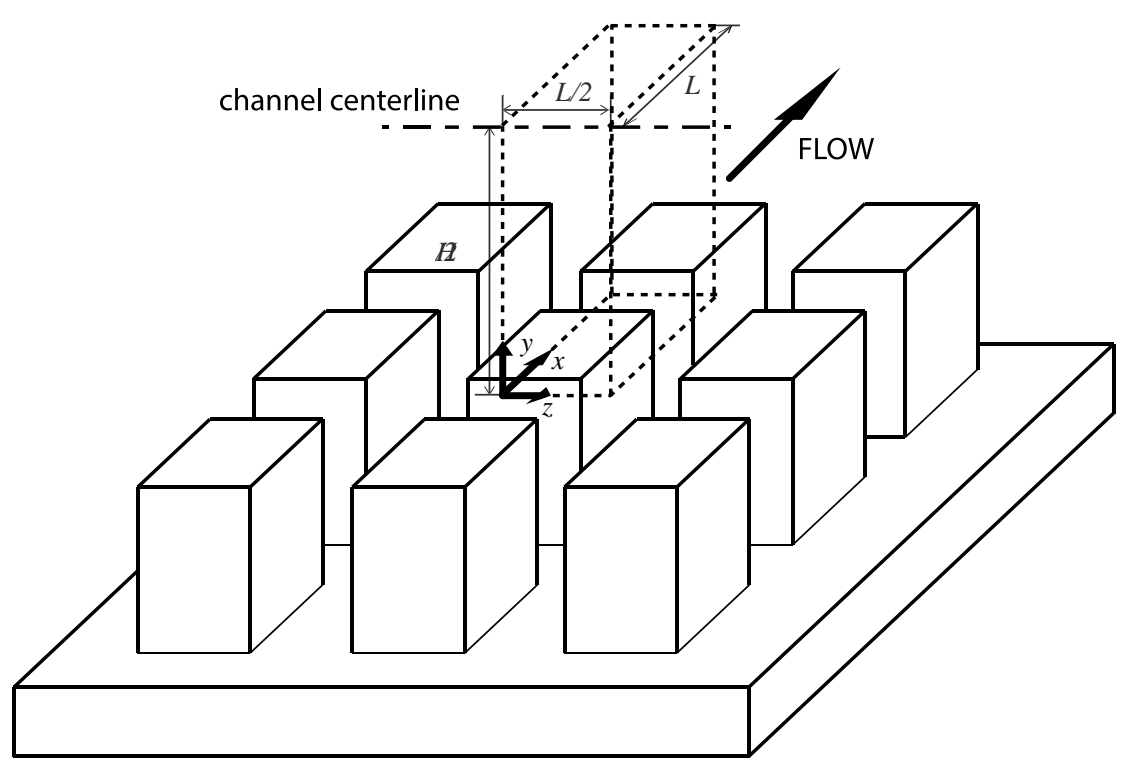

Figure 2: Schematic of scenario considered.

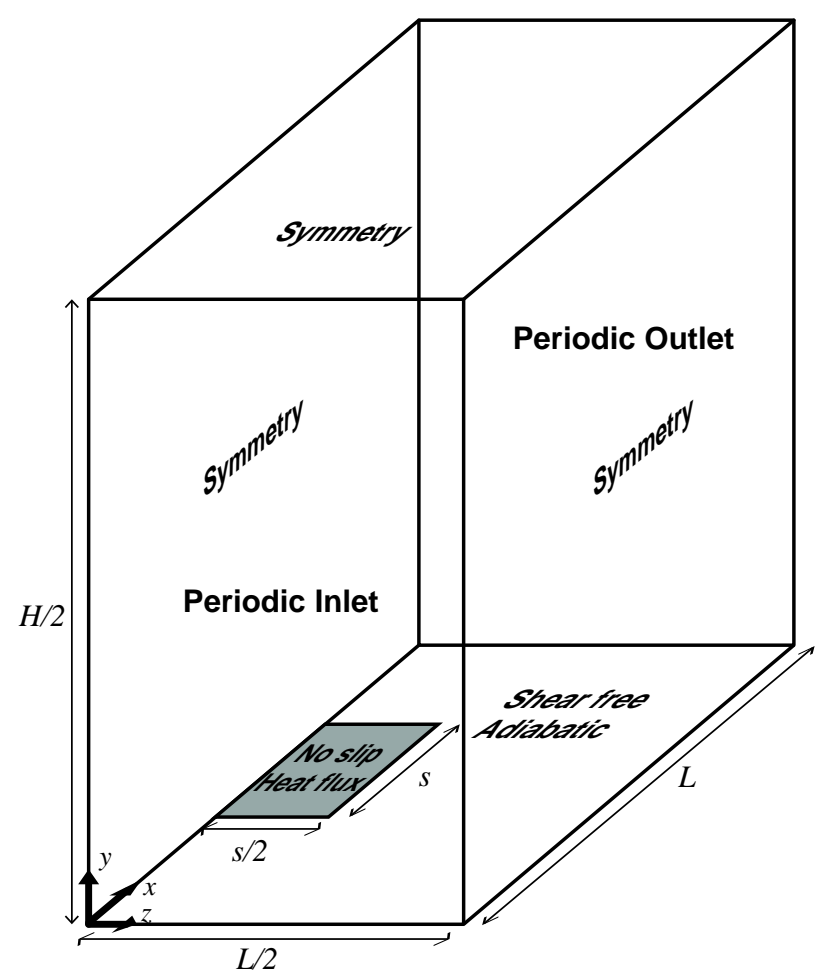

Figure 3: Computational domain and boundary conditions.
The following transformation yields periodicity in the local pressure field

$$
p(x, y, z)=-\beta x+\hat{p}(x, y, z)
$$

where $\beta$ is directly related to the mass flow rate and geometry, and $\hat{p}(x, y, z)$ describes the local pressure variations [49].

The temperature field can be transformed in a similar manner so that periodic boundary conditions for the inlet and outlet can be utilized [49]. Though the temperature field is different from module to module, the shape of the temperature profile is similar for the constant heat flux scenario considered such that [49]

$$
\begin{aligned}
& \frac{T(x+L, y, z)-T(x, y, z)}{L}= \\
& \quad \frac{T(x+2 L, y, z)-T(x+L, y, z)}{L}=\ldots=\gamma
\end{aligned}
$$

$\gamma$ is determined from an energy balance on a single module

$$
\gamma=\frac{Q}{\dot{m} c_{p} L}
$$

where $Q$ is the rate of heat addition per control volume to the fluid in the module length $L$ and $\dot{m}$ is the mass flow rate in the control volume [49]. A transformation divides the temperature field into two parts

$$
T(x, y, z)=\gamma x+\hat{T}(x, y, z)
$$

where $\hat{T}$ is periodic [49]. Substituting (10) into (5) yields

$$
\rho c_{p}(\vec{V} \cdot \nabla \hat{T}+u \gamma)=k_{f} \nabla^{2} \hat{T}
$$

where $\hat{T}$ can now be determined for the periodic computational domain depicted in Figure 3. 
STAR-CCM $+{ }^{\circledR}$ (version 10.02.010 double precision) was first used to solve the discretized continuity and momentum equations. A fully developed periodic interface was used to implement (7) within STAR-CCM+ ${ }^{\circledR}$ natively, with the periodic interfaces located at $x=0$ and $x=L$ (see Figure 3 ) where $\beta$ is iteratively determined for a specified mass flow rate $(\dot{m})$. Symmetry boundary conditions were implemented at $z=0$ and $z=L / 2$ in accordance with the infinite parallel plate assumption. Additionally, a symmetry boundary condition was used at $y=H / 2$ assuming symmetrical surface structures. At the $y=0$ boundary the following composite no-slip/shear-free boundary condition was imposed

$$
\begin{cases}\vec{V}(x, y=0, z)=0 \quad \text { if } \quad \frac{L-s}{2} \leq x \leq \frac{L+s}{2} \text { and } z \leq \frac{s}{2} \\ \left.\left(\frac{\partial u}{\partial y}+\frac{\partial v}{\partial x}\right)\right|_{y=0}=\left.\left(\frac{\partial v}{\partial z}+\frac{\partial w}{\partial y}\right)\right|_{y=0}=0 & \text { otherwise }\end{cases}
$$

A wide range of Peclet numbers $(P e)$, relative module lengths $\left(W_{m}\right)$, and solid fractions $\left(F_{s}=s^{2} / L^{2}\right)$ were explored, where the Peclet number is based on the channel hydraulic diameter $\left(D_{h}=2 H\right)$, and $s$ is the top side length of the square post (see Fig. 3). Over 200 simulations were performed in total. Initial meshes were on the order of 40,000 cells, with local refinement near the $y=0$ plane. Mass flow rate was specified according to the desired Reynolds/Peclet number and channel wall-towall spacing. All fluid properties were specified as those of water at $293 \mathrm{~K}$ (resulting in a Prandtl number of 6.96).

The convergence criteria for the hydrodynamic solution was the friction factor-Reynolds number product $(f R e)$ where $f$ is the Darcy friction factor. It can readily be shown that

$$
f R e=\frac{2 D_{h}^{2}}{\bar{u}} \beta
$$

where $\bar{u}$ is the mean fluid velocity. A simulation was deemed converged once $f R e$ changed by less than $10^{-4}$ in 1000 iterations. Once the simulation was converged, an adaptive mesh refinement technique was employed to achieve grid independence. The refinement process continued until the difference in $f R e$ between successive meshes was less than $0.1 \%$. Final meshes ranged from nominally 100,000 to $2,000,000$ cells.

After the hydrodynamic solution was obtained, the velocity data was used to solve the transformed energy equation (11). For the thermal simulation, periodic internal interfaces were used at the $x=0$ and $x=L$ planes (see Figure 3). As with the hydrodynamic domain, symmetry boundary conditions were used at the $z=0, z=L / 2$, and $y=H / 2$ planes. At the $y=0$ plane a composite constant heat flux/adiabatic boundary condition was used

$$
\left.\frac{\partial \hat{T}}{\partial y}\right|_{y=0}= \begin{cases}-q_{s}^{\prime \prime} / k_{f} & \text { if } \frac{L-s}{2} \leq x \leq \frac{L+s}{2} \text { and } z \leq \frac{s}{2} \\ 0 & \text { otherwise }\end{cases}
$$

where $q_{s}^{\prime \prime}$ is the imposed heat flux at the top of the post. The average heat flux to the domain $\left(q_{c}^{\prime \prime}=q_{s}^{\prime \prime} F_{s}\right)$ was maintained constant for all simulations. Once $\hat{T}$ is obtained, $T$ can easily be recovered via (10).

The thermal solution was deemed converged once the average Nusselt number $(\overline{N u})$ changed by less than $10^{-5}$ in 1000 iterations. It was found that adaptively refining the mesh yielded no significant change in $\overline{N u}$ if the mesh had already been refined as outlined above for the hydrodynamic solution.

Looking at the channel macroscopically there will exist some apparent slip velocity as well as apparent temperature jump at the composite interface (see Fig. 1). In order to calculate $\overline{N u}$, the following expression, rearranged from Cowley et al. [35], was used ${ }^{1}$

$$
\overline{N u}=\left(\frac{b_{t}}{L} W_{m}+\frac{17}{140}-\frac{3}{70} U_{s}+\frac{1}{210} U_{s}^{2}\right)^{-1}
$$

where $U_{s}=u_{s} / \bar{u}$ is the normalized slip velocity at the composite solid/liquid and gas/liquid interface (i.e. $y=0$ ). The temperature jump length can be readily calculated by using Fourier's law $\left(q_{c}^{\prime \prime} / k_{f}=-\left.\left(d T^{*} / d y\right)\right|_{y=0}\right)$ and (2)

$$
b_{t}=\frac{k_{f}}{q_{c}^{\prime \prime}}\left(\bar{T}_{s}-\bar{T}_{c}\right)
$$

Equation (16) can then be used with (15) to obtain $\overline{N u}$.

\section{Results and Discussion}

\subsection{Hydrodynamic Results}

Our results are first compared to prior results although our present data spans a more exhaustive range than previously explored. We then investigate the effect of Reynolds number and relative module length on the slip length.

In Fig. 4, $f R e$ is plotted as a function of Reynolds number at two separate solid fractions $\left(F_{s}=0.5\right.$ and 0.05$)$ for a fixed relative module length of 0.25 . The results are compared to the work of Cheng et al. [9], who numerically studied the effect of Reynolds number, as well as the Stokes flow case extracted from the work of Enright et al. [36]. The trend of increasing $f R e$ with Reynolds number agrees well with the work of Cheng et al. [9], though a small offset exists for the solid fraction of 0.05 . In the low Reynolds number regime our results asymptote closely to the Stokes flow solution. For this relative module length value, $f R e$ does not significantly deviate from Stokes flow until $R e \gtrsim$ 100.

In Fig. $5 f R e$ is plotted as a function of solid fraction for a fixed relative module length of 0.25 and Reynolds number of 1000 . The results are compared to a different

\footnotetext{
${ }^{1}$ This expression is equivalent to those presented by Maynes et al. [34], Enright et al. [36], and Wang [40] for a parallel plate channel with symmetric slip lengths.
} 


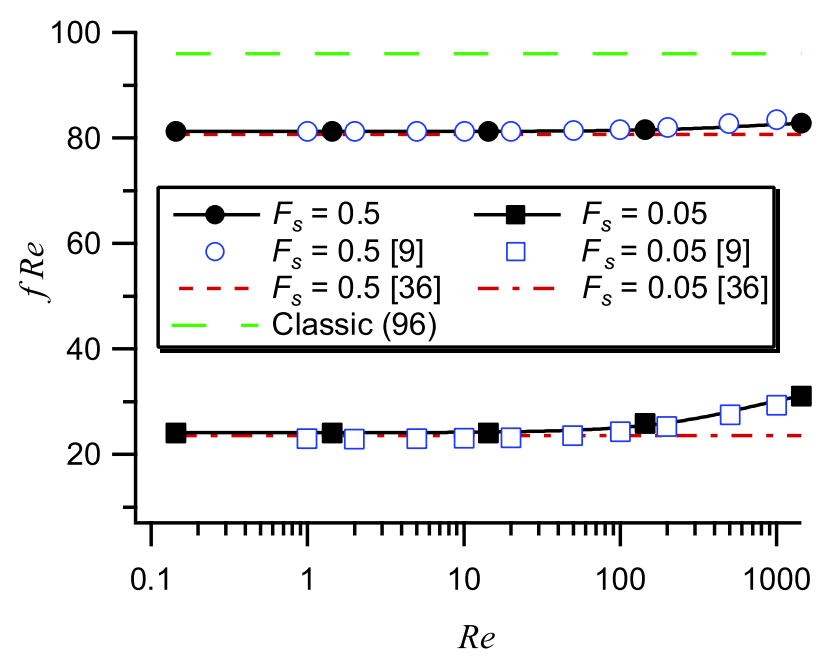

Figure 4: $f$ Re data (solid markers) is compared to the work of Cheng et al. [9] (open markers) and Enright et al. [36] (dashed and dashed dotted lines) for two solid fractions $\left(F_{s}=0.5\right.$ and 0.05$)$ as a function of Reynolds number. The circles and squares correspond to $F_{s}=0.5$ and $F_{s}=0.05$ respectively. The relative module length is fixed at 0.25 . The solid lines are splines fit through our numerical results. The long dashed line shows the classical parallel plate channel value, $f R e=96$, for reference [50].

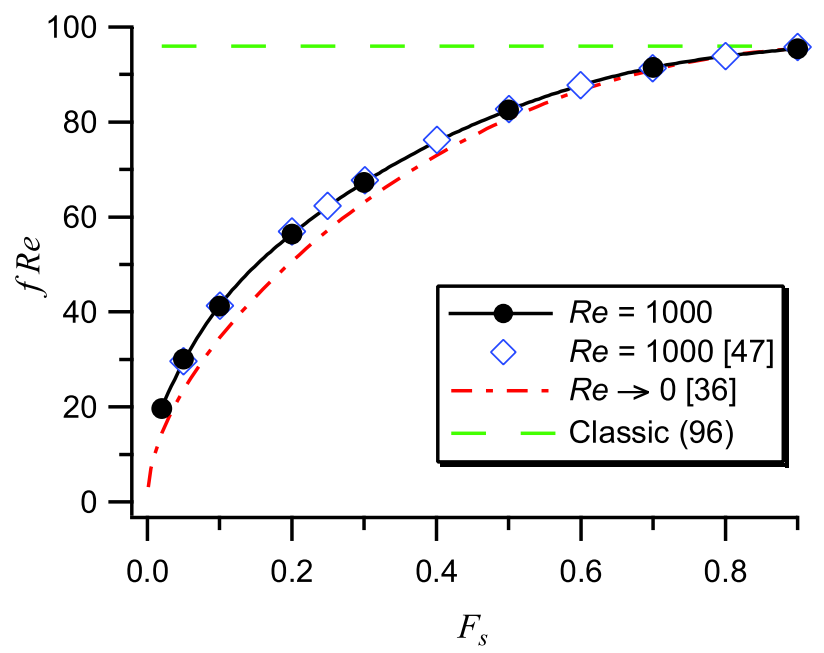

Figure 5: $f R e$ results (solid markers) are compared to the work of Cheng et al. [47] (open markers) and Enright et al. [36] (dashed dotted line) for $R e=1000$ as a function of solid fraction. Relative module length is fixed at 0.25 . The solid line is a spline fit through our numerical results. The long dashed line shows the classical parallel plate channel value, $f R e=96$, for reference [50].

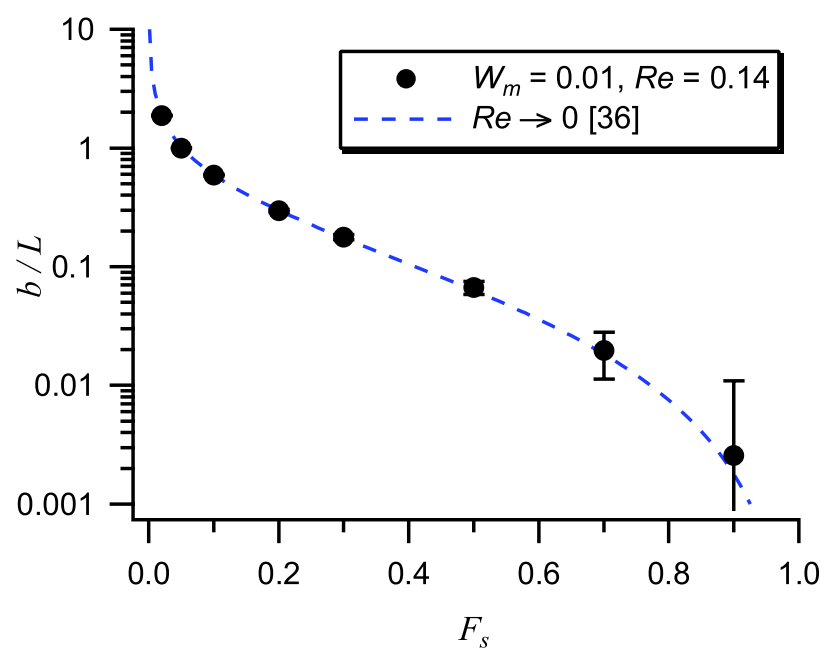

Figure 6: Slip length results (solid markers) are compared to the work of Enright et al. [36] (dashed line) for a fixed relative module length of 0.01 and Reynolds number of 0.14. Error bars are included based on the convergence criteria of $0.1 \%$ in $f R e$.

study by Cheng et al. [47] where Reynolds number effects were also included, and the Stokes flow scenario of Enright et al. [36]. At this elevated Reynolds number it can be seen that $f R e$ increases for all solid fractions in comparison to the Stokes flow case and our results have excellent agreement with the work of Cheng et al. [47]. As solid fraction approaches unity, the classical value $(f R e=96)$ is regained as expected [50].

As outlined by Davies et al. [4], the friction factorReynolds number product can be converted to the slip length for a parallel plate channel as follows

$$
\frac{b}{L}=\frac{1}{W_{m}}\left(\frac{8}{f R e}-\frac{1}{12}\right)
$$

In Fig. $6 b / L$ is compared to the Stokes flow solution of Enright et al. [36] for $R e=0.14$ and a relative module length of 0.01 and as a function of solid fraction. At this low Reynolds number and small relative module length our numerical results agree very well with those of Enright et al. [36] for all solid fractions except $F_{s}=0.9$. The large error associated with high solid fractions is due to the nature of (17); as $f R e \rightarrow 96, b / L \rightarrow 0$, and the small error associated with $f R e$ (which is $0.1 \%$ based on the convergence criteria used) propagates to a large relative error in $b / L$ (see error bars of Fig. 6). For practical applications, large solid fractions are of little interest due to their very small slip lengths and negligible effect on the flow and frictional drag.

Ybert et al. [5] proposed that $b / L$ should scale as $1 / \sqrt{F_{s}}$ in the low solid fraction limit for post structured surfaces. Several studies $[5,9,11,29,36]$ have provided the linear coefficients $(A$ and $B)$ at $R e \leq 1$ for such a scaling in the form of

$$
\frac{b}{L}=\frac{A}{\sqrt{F_{s}}}-B
$$


Table 1: Comparison of linear coefficients for (18) in the Stokes flow regime $(R e \leq 1)$.

\begin{tabular}{lll}
\hline \hline & $A$ & $B$ \\
\hline$R e=0.14, W_{m}=0.01$ & 0.3238 & 0.4295 \\
Ybert et al. [5] & 0.325 & 0.44 \\
Cheng et al. [9] & & \\
$\left(R e=1, W_{m}=0.25\right)$ & 0.336 & 0.450 \\
Ng and Wang [29] & 0.33 & 0.461 \\
Davis and Lauga [11] & 0.3323 & 0.4208 \\
Enright et al. [36] & 0.3323 & 0.4685 \\
\hline \hline
\end{tabular}

Coefficients are included in Table 1 for multiple studies as well as a those obtained from a linear regression of our data for $F_{s} \leq 0.3, R e=0.14$, and $W_{m}=0.01$. The good agreement of the $f R e$ data as well as the slip length data with several studies verifies our numerical methodology and gives confidence in our further results.

Cheng et al. [9] found that the linear scaling with $1 / \sqrt{F_{s}}$ can be extended to higher Reynolds number flows, where $A$ and $B$ become functions of the Reynolds number. We perform a linear regression in the form of (18) for each Reynolds number and relative module length combination explored for the low solid fraction region $F_{s} \leq 0.3$ ( 5 data points per regression). The regressions over all cases yield an average relative error of $1.8 \%$ with the largest errors (maximum of 11\%) occurring at the highest solid fraction of $F_{s}=0.3$. We find here that the linear scaling can be extended to higher relative module lengths as well as higher Reynolds numbers. In Fig. 7 we present several of our linear fits for $b / L$ vs. $1 / \sqrt{F_{s}}$ at various Reynolds number and relative module length combinations.

In the top panel of Fig. 7 the relative module length is fixed at 0.1 and three Reynolds numbers are shown. Also, the Stokes flow regime case $\left(R e=0.14, W_{m}=0.01\right)$ is shown in all panels for reference. It can be seen in the top panel of Fig. 7 that only at the highest Reynolds number of 1440 and smaller solid fractions (i.e. larger $1 / \sqrt{F_{s}}$ values) is there appreciable difference in the normalized slip length from the diffusion dominated solution.

In the middle panel of Fig. 7 the relative module length is fixed at 0.25 . It can be observed that the slip length now shows greater dependence on the Reynolds number. As the relative channel size decreases (i.e. increasing $W_{m}$ ) the hydrodynamic boundary layer that must redevelop over each post occupies a larger relative region of the channel and the overall flow becomes more sensitive to changes in that boundary layer. Consequently, greater Reynolds number dependency exists as $W_{m}$ increases.

This is further illustrated in the bottom panel of Fig. 7 where the relative module length is now fixed at unity. In this relatively very narrow channel, the slip length is even more affected by Reynolds number as the boundary layer occupies even more of the channel, relatively. At $R e=144$ and $F_{s}=0.02$ the normalized slip length has been reduced by more than $25 \%$ from the Stokes flow scenario and by more than $45 \%$ for $R e=1440$ and $F_{s}=0.02$. It can be
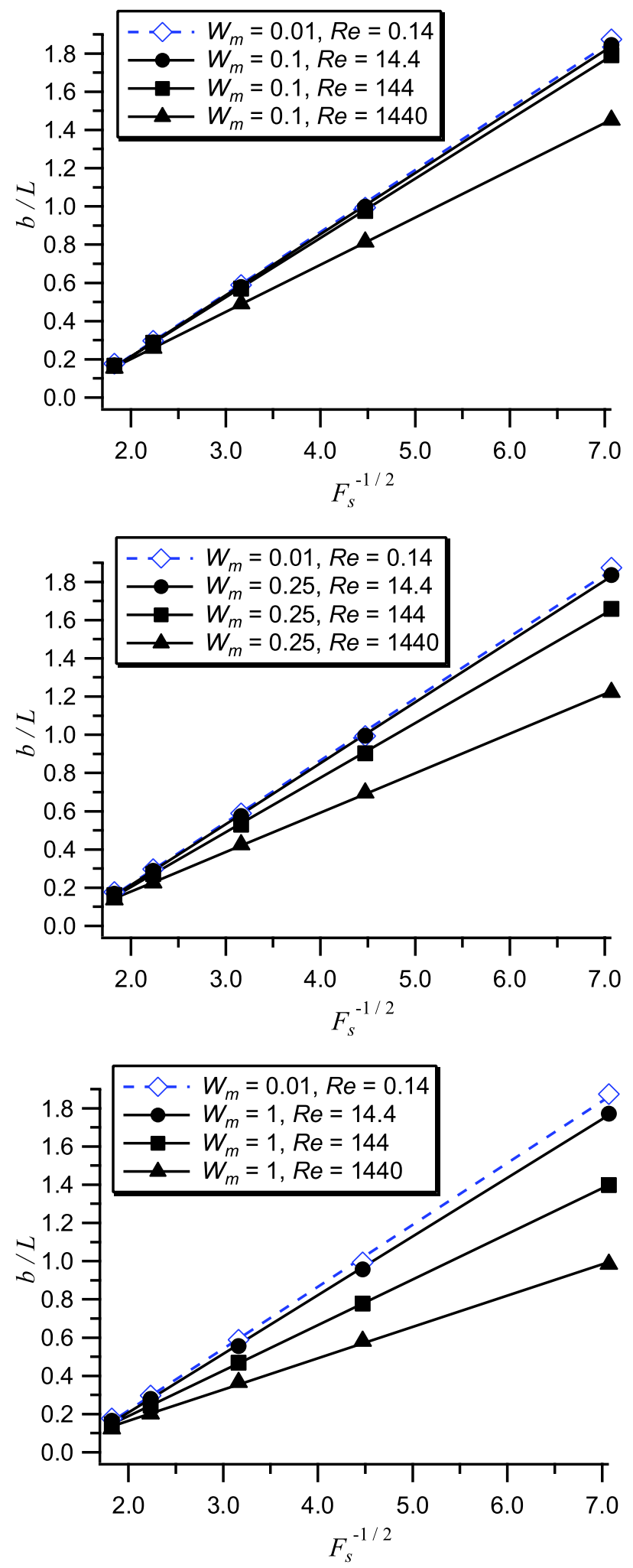

Figure 7: Normalized slip length plotted vs. $F_{s}^{-1 / 2}$ for three Reynolds numbers. Each of the three panels explores a different relative module length. Top panel: $W_{m}=0.1$. Middle panel: $W_{m}=0.25$. Bottom panel: $W_{m}=1$. Also, in each panel the Stokes regime case of $R e=0.14$ and $W_{m}=0.01$ is plotted for reference. Markers indicate numerical data and the lines are the fits through the data. 


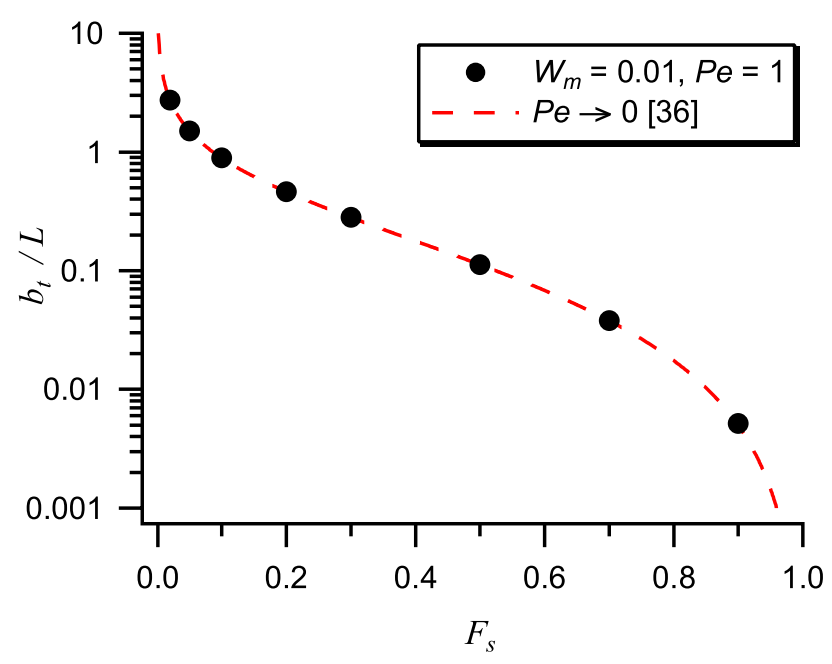

Figure 8: Temperature jump length results (solid markers) are compared to the work of Enright et al. [36] (dashed line) for a fixed relative module length of 0.01 and Peclet number of 1 .

concluded that $A$ and $B$ are functions of both Reynolds number and $W_{m}$ and that at larger relative module lengths the effect of increasing Reynolds number is magnified.

\subsection{Thermal Results}

In this section we first present the thermal results (which are the main focus of this paper) in terms of temperature jump length normalized by the module length $\left(b_{t} / L\right)$, then in terms of the average Nusselt number $(\overline{N u})$.

Enright et al. [36] recast a conduction spreading resistance solution to give the normalized temperature length for a diffusion dominated, semi-infinite scenario for posts maintained at a constant heat flux

$$
\begin{aligned}
\frac{b_{t}}{L}= & \frac{2}{\pi^{3} F_{s}}\left[\sum_{m=1}^{\infty} \frac{\sin ^{2}\left(m \pi \sqrt{F_{s}}\right)}{m^{3}}\right. \\
& \left.+\frac{1}{\pi^{2} F_{s}} \sum_{m=1}^{\infty} \sum_{n=1}^{\infty} \frac{\sin ^{2}\left(m \pi \sqrt{F_{s}}\right) \sin ^{2}\left(n \pi \sqrt{F_{s}}\right)}{m^{2} n^{2} \sqrt{m^{2}+n^{2}}}\right]
\end{aligned}
$$

In Fig. 8 we compare our results for the $P e=1$, $W_{m}=0.01$ case to (19) computed with 1000 terms for both $m$ and $n$. The agreement between our results and the analytical expression provided by Enright et al. [36] is excellent and provides verification of our numerical methods for the thermal solution. The relative difference between our numerical results and (19) is less than $0.6 \%$ for $F_{s} \leq 0.7$.

Enright et al. [36] showed that the temperature jump length for the low solid fraction region also scales as $1 / \sqrt{F_{s}}$

$$
\frac{b_{t}}{L}=\frac{C}{\sqrt{F_{s}}}-D
$$

where $C$ and $D$ are linear fit coefficients. The coefficients Enright et al. [36] obtained for a linear fit to (19) with
Table 2: Comparison of linear coefficients for (20) in the diffusion dominated regime.

\begin{tabular}{lll}
\hline \hline & $C$ & $D$ \\
\hline$P e=1, W_{m}=0.01$ & 0.4699 & 0.5930 \\
Enright et al. [36] & 0.4718 & 0.6037 \\
\hline \hline
\end{tabular}

$F_{s} \leq 0.25$ are compared in Table 2 to the coefficients we obtained by a linear regression to our $P e=1, W_{m}=0.01$, $F_{s} \leq 0.2$ data. The differences between our coefficients and those of Enright et al. [36] are $0.4 \%$ and $1.8 \%$ for $C$ and $D$, respectively.

Similar to the hydrodynamic solution, we perform a linear regression in the form of (20) for each Peclet number/relative module length combination explored. We confine our regressions to the low solid fraction range $F_{s} \leq 0.2$ (4 data points per regression) and find that the linear scaling extends very well to both higher Peclet numbers and relative module lengths. The regressions over all cases yield an average relative error of $0.7 \%$ with the largest errors (maximum of $4.4 \%$ ) occurring at the highest solid fraction of $F_{s}=0.2$. In Fig. 9 we present several of our linear fits for $b_{t} / L$ vs. $1 / \sqrt{F_{s}}$ at various Peclet number and relative module length combinations (the same combinations as in Fig. 7) to illustrate the effects of increasing $\mathrm{Pe}$ and $W_{m}$.

In the top panel of Fig. 9 the relative module length is fixed at 0.1 and three Peclet numbers are shown. Also, the Stokes flow regime case $\left(W_{m}=0.01, P e=1\right)$ is shown in all panels for reference. It can be seen that the temperature jump length is more affected by the increasing Reynolds/Peclet number than the hydrodynamic slip length (compare top panel of Fig. 7). This is mainly due to the Peclet number being higher than the Reynolds number for each case because the Prandtl number for water is nominally 7 in our simulations. Thus the ratio of inertia to thermal diffusion is higher than the ratio of inertia to viscous diffusion in all cases.

In the middle panel of Fig. 9 the relative module length is fixed at 0.25 . The effect of increasing Peclet number becomes magnified with larger relative module length, and at a Peclet number of 100 and the smallest solid fraction there is deviation from the diffusion dominated case of nominally $10 \%$. At the lowest solid fraction for the $P e=$ 10000 case, the temperature jump length is reduced by more than $50 \%$ from the diffusion dominated case. As Pe increases the increased inertia allows relatively cooler fluid to be transported closer to the heated tops of the posts, which increases heat transfer and reduces the temperature jump length.

In the bottom panel of Fig. 9 the relative module length is fixed at 1 . We have also included the $W_{m}=1, P e=1$ case to illustrate an interesting effect. We see that even at this low Peclet number case there is some deviation from the diffusion dominated case. As the symmetric posts from each side of the channel become close to one another, the 

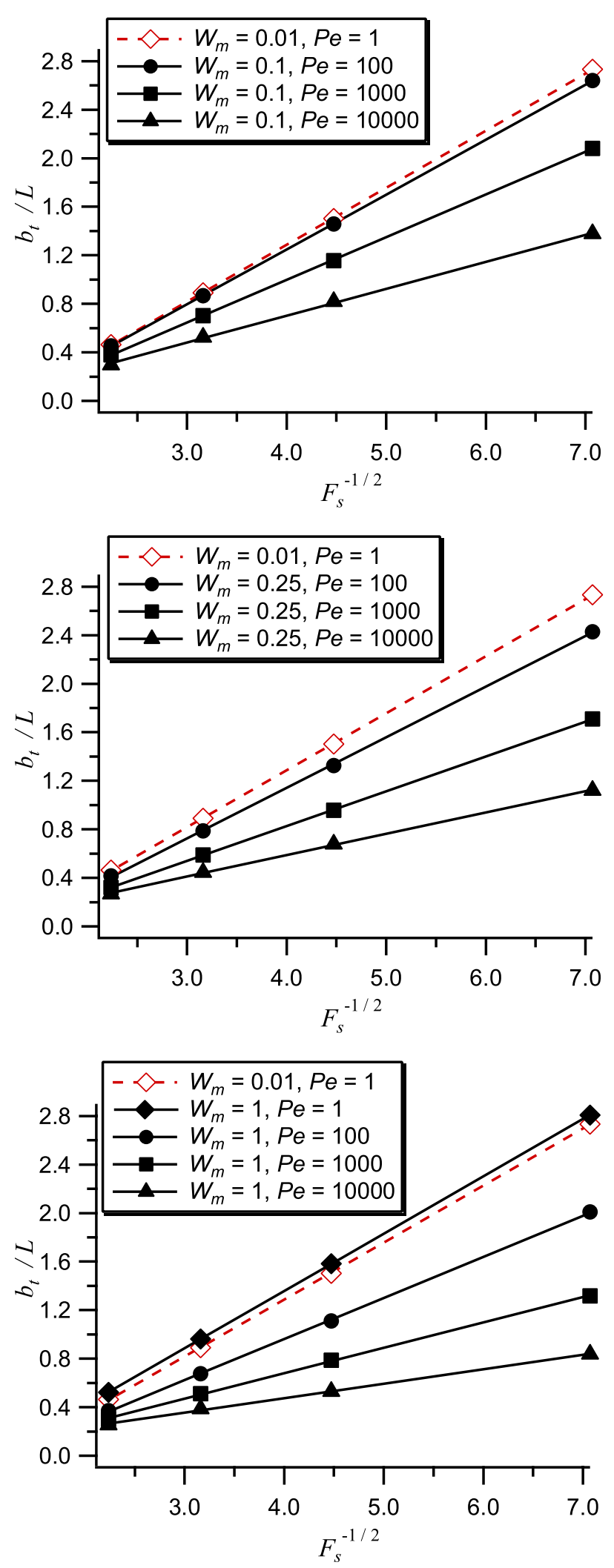

Figure 9: Normalized temperature jump length plotted vs. $F_{s}^{-1 / 2}$ for three $P e$ numbers (except bottom panel where four $P e$ are shown). Each of the three panels explores a different relative module length. Top panel: $W_{m}=0.1$. Middle panel: $W_{m}=0.25$. Bottom panel: $W_{m}=1$. Also, in each panel the Stokes flow diffusion dominated case of $P e=1$ and $W_{m}=0.01$ is plotted for reference. Markers indicate numerical data and the lines are the fits through the data. temperature gradient in the $y$ direction will be reduced. This confinement effect limits the heat transfer and consequently the temperature jump length is increased slightly with respect to the diffusion dominated case. As $P e$ increases, the inertia effects prevail and increase the heat transfer which reduces the temperature jump length with respect to the diffusion dominated case.

We now present the thermal results in terms of the average Nusselt number which allows for direct comparison to a classical smooth wall channel as well as between different relative module lengths. In Fig. 10 we show the average Nusselt number as a function of $\sqrt{F_{s}}$ for three relative module lengths $(0.01,0.1$, and 1$)$ and three Peclet numbers $(1,100,10000)$. The corresponding Stokes/diffusion dominated solution, extracted from the work of Enright et al. [36], is shown for each relative module length ${ }^{2}$. Also, the classic smooth wall channel value (8.235) is shown for reference [51].

In the top panel of Fig. 10 the relative module length is fixed at 0.01 . The $P e=1, P e=100$, and diffusion dominated curves are nearly indistinguishable. Also, only a very slight deviation can be seen for the $P e=10000$ curve at the very smallest solid fractions. This means that at this small relative module length, inertia effects are negligible on the overall heat transfer and the diffusion dominated solution of Enright et al. [36] can be used. Similar to the hydrodynamics, this occurs for large relative channels because $\vec{V} \rightarrow 0$ near the surface features, thus a local Stokes flow exists in close proximity to the features and thermal diffusion dominates in close proximity to the interface. As expected, the overall heat transfer decreases with decreasing solid fraction since the insulating gas/liquid interface comprises more of the composite interface. At the largest solid fractions the classical value of 8.235 is approached, since, by definition a solid fraction of unity is a classical smooth channel. Overall, the heat transfer does not deviate much from a classic smooth wall channel for this relative module length. This occurs because the posts are small relative to the channel size and they can only exert a small influence on the overall flow and heat transfer.

In the middle panel of Fig. 10 the relative module length is fixed at 0.1. Similar to the $W_{m}=0.01$ case, the $P e=1, P e=100$, and diffusion dominated curves are nominally the same. However, the $P e=10000$ curve now shows significant difference from the diffusion dominated case. For this relative module length, once advection becomes strong enough near the composite interface (i.e. high enough $\mathrm{Pe}$ ) it can increase the overall convective heat transfer. This occurs because the higher inertial forces cause the thermal boundary layer that redevelops on the solid/liquid interface in each module to become thinner, thus increasing the temperature gradient in the $y$ direction

\footnotetext{
${ }^{2}$ Note that the hydrodynamic and thermal slip lengths of Enright et al. [36] were obtained for a diffusion dominated scenario. When calculating the average Nusselt number with (15), advection is accounted for.
} 

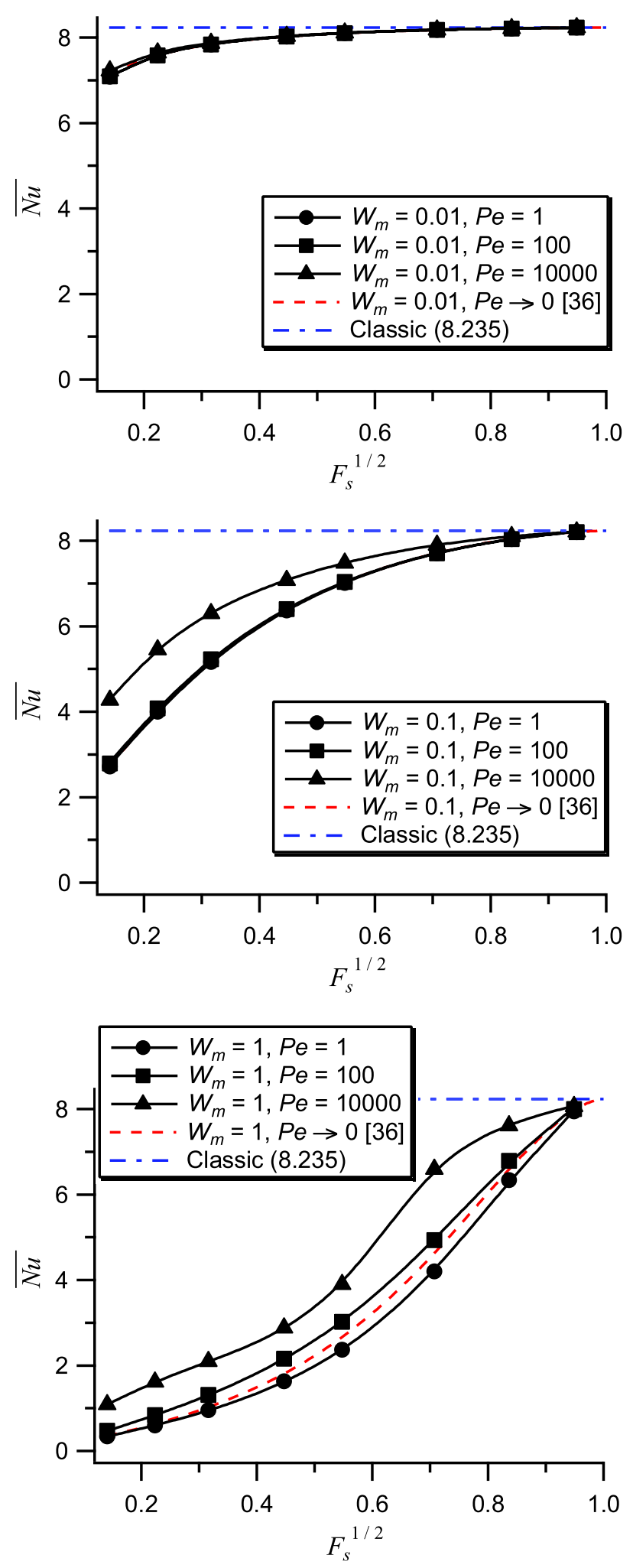

Figure 10: Average Nusselt number plotted vs. $F_{s}^{1 / 2}$ for three Peclet numbers $(1,100,10000)$. Each of the three panels explores a different relative module length. Top panel: $W_{m}=0.01$. Middle panel: $W_{m}=0.1$. Bottom panel: $W_{m}=1$. Our results are represented by the solid markers and the solid lines are splines through the data. In each panel the Stokes flow diffusion dominated case extracted from the work of Enright et al. [36] is shown by the dashed line. The dashed-dotted line shows the classical value of 8.235 for reference [51].

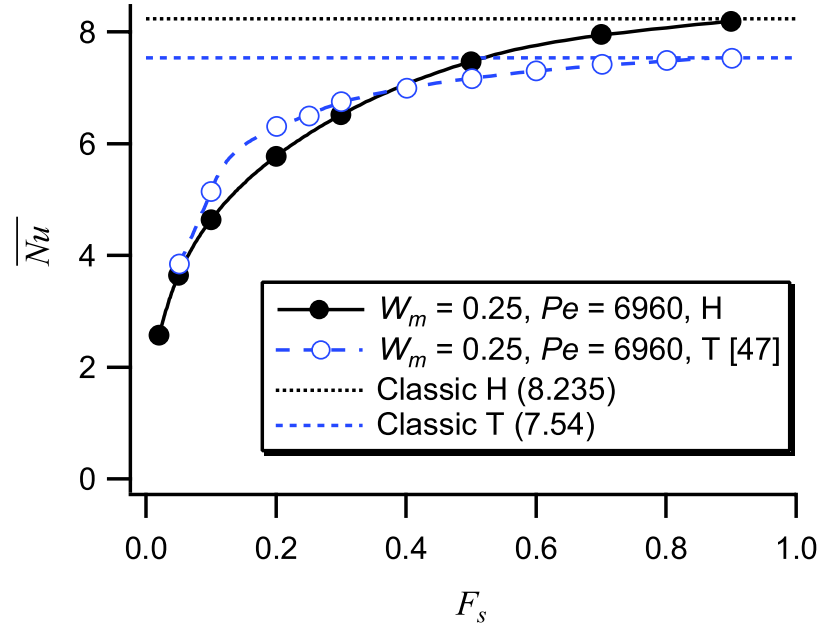

Figure 11: Average Nusselt number vs. solid fraction for $P e=6963$ and $W_{m}=0.25$. Our data for a constant heat flux boundary condition $(\mathrm{H})$ is shown by the solid markers with the solid line. The data of Cheng et al. [47] for a constant temperature boundary condition (T) is shown by the open markers with the dashed line. Where the lines are splines through the data. The classical smooth channel values of 8.235 and 7.54 [51] for the constant heat flux and constant temperature boundary conditions are shown by the fine dotted and fine dashed lines respectively.

at the interface which increases the overall heat transfer when compared to lower Peclet numbers. When compared to the $W_{m}=0.01$ case, the average Nusselt number deviates more from the classical channel case. This affirms that as the module length approaches the scale of the channel size (i.e. increasing $W_{m}$ ), SH surfaces cause a more pronounced reduction on the overall heat transfer.

In the bottom panel of Fig. 10 the relative module length is fixed at 1 . At this large relative module length we observe larger deviations from the diffusion dominated case with increasing Peclet number. Even at $P e=1$ there is some deviation from the diffusion dominated results of Enright et al. [36]. This is likely caused by the very small relative channel spacing (i.e. confinement effects discussed earlier) since the work of Enright et al. [36] assumed a semi-infinite domain and this is no longer the case. It can be concluded that as the relative module length increases, inertial effects become more influential on the thermal transport. Also, it can be concluded that as $W_{m}$ increases the effect of the SH walls on the overall heat transfer reduction is greater. At the smallest solid fraction and lowest Peclet number $\left(F_{s}=0.02, P e=1\right)$ the average Nusselt number is only $4.2 \%$ of the classical channel value.

For completeness we explore the difference between a constant heat flux and a constant temperature boundary condition. In Fig. 11 we show $\overline{N u}$ vs. $F_{s}$ for our results, where the solid/liquid interface was held at a constant heat flux, and for the results of Cheng et al. [47], where the solid/liquid was held at a constant temperature. The relative module length is 0.25 and the Peclet number is 6963 $(R e=1000)$, where they [47] also used water at $293 \mathrm{~K}$ as their working fluid. The overall trend is very similar for 
both thermal boundary conditions. As $F_{s} \rightarrow 1$ each curve approaches its respective smooth wall limit. For solid fractions less than 0.4 the constant temperature case has a slightly higher Nusselt number, whereas after 0.4 this behavior reverses and the constant heat flux case is higher. Additional relative module lengths are not available for comparison, but we can conclude for the $W_{m}=0.25$, $P e=6963$ case that the thermal boundary condition does not largely change the overall thermal transport behavior.

It should be noted that while the meniscus was assumed to be flat and adiabatic, effects at the meniscus such as curvature, heat transfer, and mass transfer can affect both the hydrodynamic and thermal results $[7,10,37,38]$. Solving for the local meniscus shape in pressure driven flow would require the local pressure differential across the liquid/gas interface for the entire length of the channel; which would require a specific channel geometry. This would not allow for periodicity in the boundary conditions and was beyond the scope of this study.

\subsection{Regime Mapping}

For practical use we have provided a mapping, valid over the range of parameters explored, depicting where our results differ by $5 \%$ or more from the Stokes flow/semiinfinite solutions given by Enright et al [36]. $\hat{F}_{s, f R e}$, defined as the lowest value of solid fraction where our $f R e$ results are still within $5 \%$ of the Stokes flow solution, is plotted vs. Reynolds number for 5 relative module lengths in the left panel of Fig. $12 \hat{F}_{s, f R e}$. In practice, one can compute the relative module length and Reynolds number for a given $\mathrm{SH}$ post channel, then, if the solid fraction of the $\mathrm{SH}$ channel is on or above the corresponding $W_{m}$ curve the Stokes flow solution of Enright et al. [36] can be utilized to determine $f R e$ with $\leq 5 \%$ error. It should be noted that for $W_{m} \leq 0.025$ one may neglect inertia effects on $f R e$, regardless of solid fraction or Reynolds number. In general, the region where the Stokes flow solution can be used becomes more restricted as relative module length and Reynolds number increase.

Similarly, in the right panel of Fig. $12 \hat{F}_{s, N u}$ is plotted vs. Reynolds number for the 5 relative module lengths explored, where $\hat{F}_{s, N u}$ is defined as the lowest value of solid fraction where our $\overline{N u}$ results are still within $5 \%$ of the Stokes flow solution. In practice, one can compute the relative module length and Peclet number for a given $\mathrm{SH}$ post channel, then, if the solid fraction of the SH channel is on or above the corresponding $W_{m}$ curve the Stokes flow solution of Enright et al. [36] can be utilized to determine $\overline{N u}$ with $\leq 5 \%$ error. For $\overline{N u}$, inertia may be neglected when $W_{m} \leq 0.01$, regardless of solid fraction or Peclet number; this is affirmed in the top panel of Fig. 10. Regions where the Stokes flow solution applies to $\overline{N u}$ is more limited than $f R e$. As discussed previously this is mainly due to the Prandtl number being greater than unity for the present study. The $W_{m}=1, P e=1$ case does not follow the same general trend as the other relative module length curves due to the confinement effects discussed earlier and the semi-infinite assumption of the Stokes flow solution no longer being accurate.

We have also provided Table 3 and Table 4 to facilitate calculation of $f R e$ and $\overline{N u}$ for low solid fractions when outside of the Stokes flow regime. Table 3 contains the linear fit coefficients $A$ and $B$ for calculation of $b / L$ from (18) for $F_{s} \leq 0.3$. Using the obtained slip length one can then solve for $f R e$ from (17).

Table 4 contains the linear fit coefficients $C$ and $D$ for calculation of $b_{t} / L$ from (20) for $F_{s} \leq 0.2$. For calculation of $\overline{N u}$ one needs both $b / L$ and $b_{t} / L$. Maynes and Crockett [34] provide an expression to convert between $b / L$ and $U_{s}$

$$
U_{s}=\left(1+\frac{L}{b} \frac{1}{W_{m} 12}\right)^{-1}
$$

By utilizing (21), (15) can be used to calculate $\overline{N u}$.

Note that the regime where the Stokes flow solutions are valid to within $5 \%$ incorporates the majority of realizable scenarios. This is due to the increase in required driving pressure for small channels relative to the feature spacing (i.e. large relative module lengths) and high Reynolds/Peclet number flows. The meniscus would not be able to support such large pressures (i.e. Laplace pressure would be exceeded) and the channel would wet and no longer be in the Cassie-Baxter state. Liquid metals may be able to support larger pressures due to their increased surface tension. However, due to their low Prandtl numbers, the Peclet number would stay low enough throughout most of the laminar regime such that the Stokes flow solution would remain valid regardless.

\subsection{Effect of Prandtl Number}

Water was used as the working fluid for all previous results, thus the thermal results are strictly valid only for a Prandtl number of nominally 7 . In order to investigate the effects of Prandtl number, two additional Prandtl numbers were used (0.07 and 700) when solving for the thermal solution. The additional cases were performed for two relative module lengths ( 0.025 and 0.25$)$, three solid fractions $(0.3$, 0.05 and 0.02$)$ and spanned three Peclet numbers $(1,100$, and 10000).

These alternate $\operatorname{Pr}$ results are presented in the form of temperature jump length in Fig. 13 where they are compared to the corresponding linear fits for $\mathrm{Pr}=7$. It can be seen in the left panel of Fig. 13 that for a relative module length of 0.025 , varying the Prandtl number has negligible effect on the temperature jump length. For small relative module lengths the Peclet number (along with solid fraction and relative module length) fully encompasses any $\mathrm{Pr}$ effects on the thermal transport behavior.

In the right panel of Fig. 13 a larger relative module length of 0.25 is explored. For $P e<100$ we see that, again, the Prandtl number has minimal effect on the temperature jump length. However, for the largest Peclet number of 10000 explored in this study there is a notable difference between the $\operatorname{Pr}=700$ and $\operatorname{Pr}=7$ results. For the 

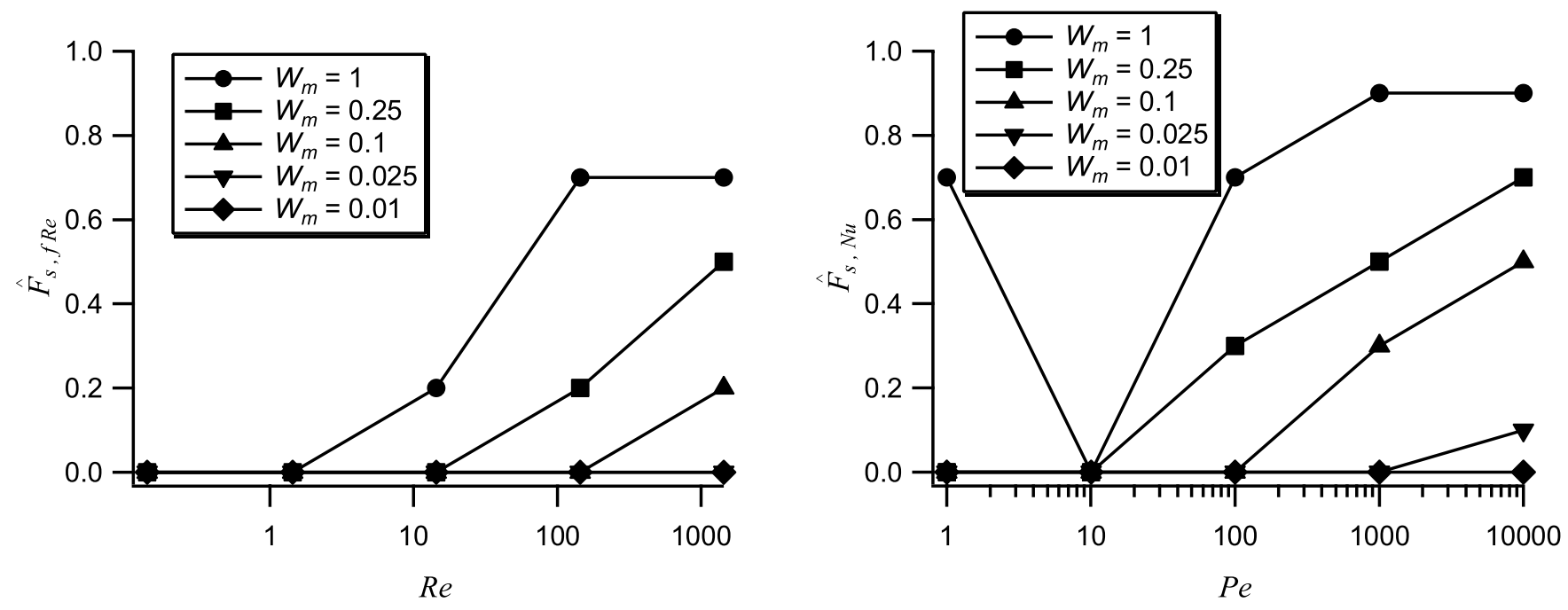

Figure 12: Left panel: Cutoff solid fraction for fRe being within $5 \%$ of the Stokes flow solution plotted vs. Re for 5 relative module lengths. Right panel: Cutoff solid fraction for $\overline{N u}$ being within $5 \%$ of the Stokes flow solution plotted vs. Pe for 5 relative module lengths.

Table 3: Coefficients $A$ and $B$ for the linear fits for $b / L$. $A$ is on top and $B$ is below in parenthesis. Valid for use with (18) for $F_{s} \leq 0.3$.

\begin{tabular}{ccccccc}
\hline \hline & & & \multicolumn{5}{c}{$\boldsymbol{W}_{\boldsymbol{m}}$} \\
\hline & & $\mathbf{0 . 0 1}$ & $\mathbf{0 . 0 2 5}$ & $\mathbf{0 . 1}$ & $\mathbf{0 . 2 5}$ & $\mathbf{1}$ \\
& \multirow{4}{*}{$\mathbf{1}$} & 0.3238 & 0.3211 & 0.3204 & 0.3201 & 0.3192 \\
& & $(0.4295)$ & $(0.4255)$ & $(0.4265)$ & $(0.4263)$ & $(0.4268)$ \\
& \multirow{4}{*}{$\mathbf{1 . 4 4}$} & 0.3238 & 0.3211 & 0.3204 & 0.3201 & 0.3190 \\
& & $(0.4295)$ & $(0.4255)$ & $(0.4265)$ & $(0.4263)$ & $(0.4266)$ \\
& \multirow{4}{*}{$\mathbf{1 4 . 4}$} & 0.3238 & 0.3211 & 0.3202 & 0.3187 & 0.3071 \\
& \multirow{4}{*}{$\mathbf{1 4 4}$} & $(0.4295)$ & $(0.4255)$ & $(0.4261)$ & $(0.4240)$ & $(0.4068)$ \\
& \multirow{4}{*}{$\mathbf{1 4 4 0}$} & 0.3238 & 0.3208 & 0.3101 & 0.2859 & 0.2386 \\
& $(0.4295)$ & $(0.4249)$ & $(0.4061)$ & $(0.3684)$ & $(0.2885)$ \\
& & 0.3166 & 0.3065 & 0.2471 & 0.2066 & 0.1638 \\
& $(0.4088)$ & $(0.3948)$ & $(0.2935)$ & $(0.2339)$ & $(0.1635)$ \\
\hline \hline
\end{tabular}

Table 4: Coefficients $C$ and $D$ for the linear fits for $b_{t} / L . C$ is on top and $D$ is below in parenthesis. Valid for use with $(20)$ for $F_{s} \leq 0.2$.

\begin{tabular}{|c|c|c|c|c|c|c|}
\hline & & & & $W_{m}$ & & \\
\hline \multirow{11}{*}{$P e$} & \multirow{3}{*}{1} & 0.01 & 0.025 & 0.1 & 0.25 & 1 \\
\hline & & 0.4699 & 0.4695 & 0.4694 & 0.4692 & 0.4725 \\
\hline & & $(0.5930)$ & $(0.5920)$ & $(0.5914)$ & $(0.5876)$ & $(0.5322)$ \\
\hline & \multirow{2}{*}{10} & 0.4699 & 0.4695 & 0.4692 & 0.4670 & 0.4470 \\
\hline & & $(0.5930)$ & $(0.5920)$ & $(0.5909)$ & $(0.5851)$ & $(0.5334)$ \\
\hline & \multirow{2}{*}{100} & 0.4699 & 0.4690 & 0.4524 & 0.4171 & 0.3389 \\
\hline & & $(0.5930)$ & $(0.5908)$ & $(0.5618)$ & $(0.5270)$ & $(0.3941)$ \\
\hline & \multirow{2}{*}{1000} & 0.4678 & 0.4441 & 0.3519 & 0.2869 & 0.2092 \\
\hline & & $(0.5876)$ & $(0.5353)$ & $(0.4111)$ & $(0.3213)$ & $(0.1574)$ \\
\hline & \multirow{2}{*}{10000} & 0.4159 & 0.3323 & 0.2222 & 0.1756 & 0.1188 \\
\hline & & $(0.4734)$ & $(0.3458)$ & $(0.1867)$ & $(0.1156)$ & $(0.0007)$ \\
\hline
\end{tabular}



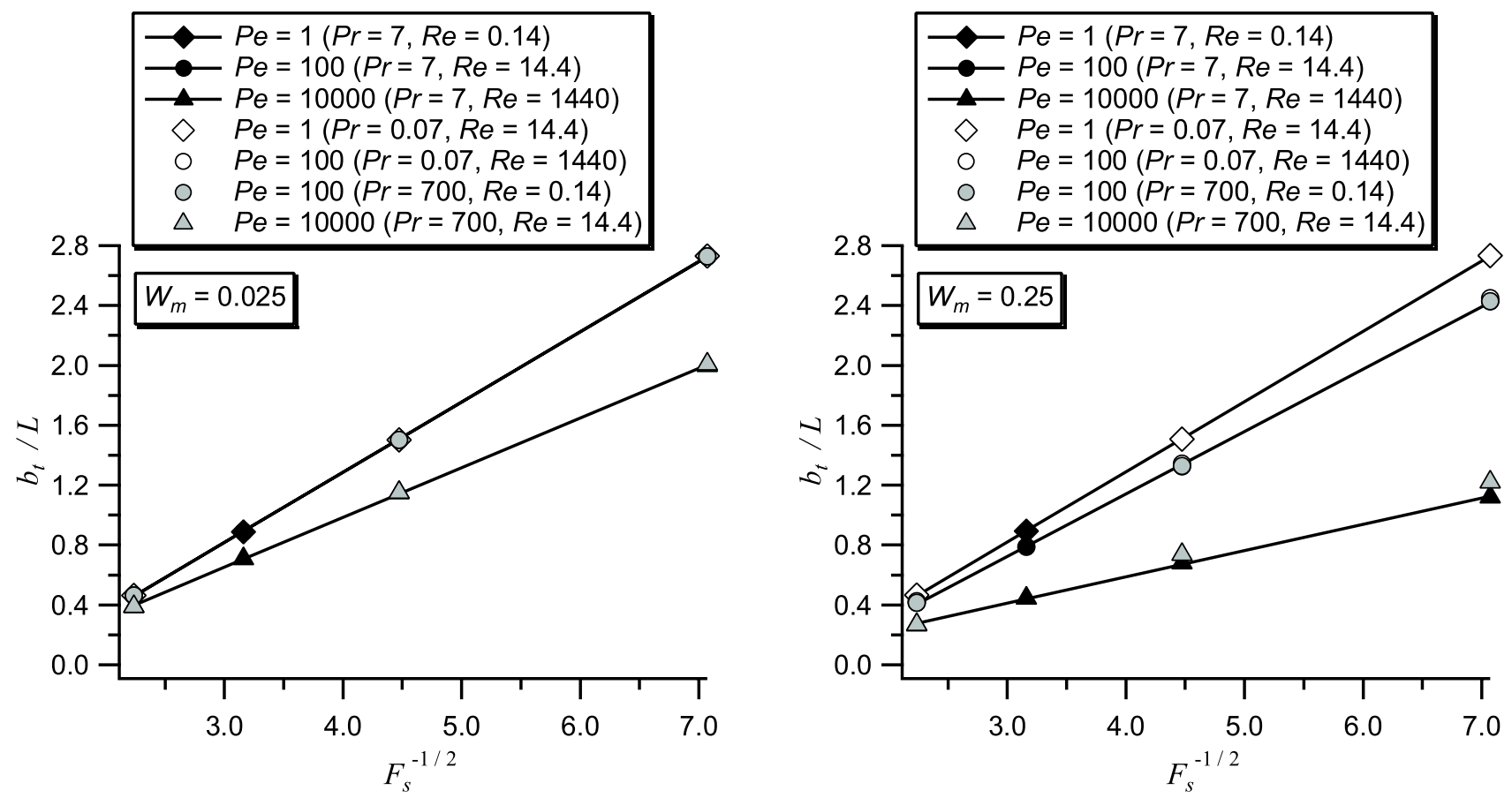

Figure 13: Normalized temperature jump length plotted vs. $F_{s}^{-1 / 2}$ for various Prandtl number and Reynolds number combinations. The $\operatorname{Pr}=0.07$ (white markers) and $\operatorname{Pr}=700$ (gray markers) results are compared to the linear fits obtained for $\operatorname{Pr}=7$ (black lines with black markers). Left panel: $W_{m}=0.025$. Right panel: $W_{m}=0.25$. The marker shape corresponds to the Peclet number.

smaller relative channel size (larger $W_{m}$ ), the hydrodynamic behavior is more sensitive to the Reynolds number as discussed in Section 3.1. Thus the velocity profile for the $\operatorname{Pr}=7$ case where $R e=1440$ is much different when compared to the $\operatorname{Pr}=700$ case where $R e=14.4$. It can be seen that this causes the temperature jump length to be slightly higher for the $\operatorname{Pr}=700$ case relative to the $\operatorname{Pr}=7$ case $\left(9 \%\right.$ higher for $\left.F_{s}=0.02\right)$.

Overall, the correlations presented in Table 4 are valid for Prandtl numbers other than 7 for a large range of parameters studied. For low $\mathrm{Pr}$, the correlations work well for the entire range of parameters explored. However, for large relative module lengths and large Prandtl numbers the presented correlations begin to deviate, though they may be used as an initial approximation.

\section{Conclusion}

Fully developed flow in a parallel plate $\mathrm{SH}$ microchannel was numerically explored in this paper. The SH surface structure was comprised of a square array of square posts. The solid/liquid interface was maintained at a constant heat flux and the air/liquid interface was assumed to be adiabatic and idealized as flat. The hydrodynamic results were presented in terms of the friction factor-Reynolds number product and hydrodynamic slip length, and the thermal results were presented in terms of the average Nusselt number and temperature jump length.

Overall, the convective heat transfer was found to be reduced in all cases for the $\mathrm{SH}$ channels when compared to a classic smooth walled channel. This work specifically addressed the effects of inertia on the thermal transport and explored a more exhaustive range of parameters than previously studied. Interestingly, for low relative module lengths, the diffusion dominated Stokes flow solutions of Enright et al. [36] agreed very well with our results regardless of Peclet number. Only when the relative module length was large enough did increasing Peclet number affect the thermal transport. A map delineating where the Stokes flow solution is valid for $f R e$ and $\overline{N u}$ is provided. In conclusion, the effect of inertia on thermal transport for post structured SH surfaces is manifest for large relative module lengths and large Peclet numbers.

\section{Acknowledgment}

This research was supported by the National Science Foundation (NSF) (Grant No. CBET-1235881). We gratefully acknowledge the Fulton Supercomputing Lab for use of their computational resources.

\section{References}

[1] A. B. D. Cassie, S. Baxter, Wettability of porous surfaces, Trans. Faraday Soc. 40 (1944) 546-551.

[2] K.-Y. Law, Definitions for hydrophilicity, hydrophobicity, and superhydrophobicity: Getting the basics right, The Journal of Physical Chemistry Letters 5 (4) (2014) 686-688.

[3] J. Ou, J. P. Rothstein, Direct velocity measurements of the flow past drag-reducing ultrahydrophobic surfaces, Physics of Fluids 17 (10) (2005) 103606-1-10. 
[4] J. Davies, D. Maynes, B. W. Webb, B. Woolford, Laminar flow in a microchannel with superhydrophobic walls exhibiting transverse ribs, Physics of Fluids 18 (8) (2006) 087110-1-11.

[5] C. Ybert, C. Barentin, C. Cottin-Bizonne, P. Joseph, L. Bocquet, Achieving large slip with superhydrophobic surfaces: Scaling laws for generic geometries, Physics of Fluids 19 (123601) (2007) 123601-1-10.

[6] D. Maynes, K. Jeffs, B. Woolford, B. W. Webb, Laminar flow in a microchannel with hydrophobic surface patterned microribs oriented parallel to the flow direction, Physics of Fluids 19 (9) (2007) 093603-1-12.

[7] A. M. J. Davis, E. Lauga, Geometric transition in friction for flow over a bubble mattress, Physics of Fluids 21 (2009) 011701$1-4$.

[8] B. Woolford, D. Maynes, B. Webb, Liquid flow through microchannels with grooved walls under wetting and superhydrophobic conditions, Microfluid Nanofluid 7 (2009) 121-135.

[9] Y. P. Cheng, C. J. Teo, B. C. Khoo, Microchannel flows with superhydrophobic surfaces: Effects of reynolds number and pattern width to channel height ratio, Physics of Fluids 21 (2009) 122004-1-12.

[10] C. J. Teo, B. C. Khoo, Flow past superhydrophobic surfaces containing longitudinal grooves: effects of interface curvature, Microfluid Nanofluid 9 (2010) 499-511.

[11] A. M. J. Davis, E. Lauga, Hydrodynamic friction of fakirlike superhydrophobic surfaces, Journal of Fluid Mechanics 661 (2010) 402-411.

[12] J. P. Rothstein, Slip on superhydrophobic surfaces, Annu. Rev. Fluid Mech. 42 (2010) 89-109.

[13] R. J. Daniello, N. E. Waterhouse, J. P. Rothstein, Drag reduction in turbulent flows over superhydrophobic surfaces, Physics of Fluids 21 (8) (2009) 085103-1-9.

[14] M. B. Martell, J. B. Perot, J. P. Rothstein, Direct numerical simulations of turbulent flows over superhydrophobic surfaces, Journal of Fluid Mechanics 620 (2009) 31-41.

[15] B. Woolford, J. Prince, D. Maynes, B. W. Webb, Particle image velocimetry characterization of turbulent channel flow with rib patterned superhydrophobic walls, Physics of Fluids 21 (8) (2009) 085106-1-12.

[16] K. Jeffs, D. Maynes, B. W. Webb, Prediction of turbulent channel flow with superhydrophobic walls consisting of micro-ribs and cavities oriented parallel to the flow direction, International Journal of Heat and Mass Transfer 53 (4) (2010) 786-796.

[17] D. Bartolo, F. Bouamrirene, É. Verneuil, A. Buguin, P. Silberzan, S. Moulinet, Bouncing or sticky droplets: Impalement transitions on superhydrophobic micropatterned surfaces, Europhysics Letters 74 (2) (2006) 229-305.

[18] M. Reyssat, A. Pépin, F. Marty, Y. Chen, D. Quéré, Bouncing transitions on microtextured materials, Europhysics Letters 74 (2) (2006) 306-312.

[19] Z. Wang, C. Lopez, A. Hirsa, N. Koratkar, Impact dynamics and rebound of water droplets on superhydrophobic carbon nanotube arrays, Applied Physics Letters 91 (2) (2007) 23105-1-3.

[20] J. Pearson, D. Maynes, B. Webb, Droplet impact dynamics for two liquids impinging on anisotropic superhydrophobic surfaces, Experiments in Fluids 53 (3) (2012) 603-618.

[21] A. L. Yarin, Drop impact dynamics: Splashing, spreading, receding, bouncing ..., Annual Review of Fluid Mechanics 38 (1) (2006) 159-192.

[22] D. Maynes, M. Johnson, B. W. Webb, Free-surface liquid jet impingement on rib patterned superhydrophobic surfaces, Physics of Fluids 23 (5) (2011) 052104-1-10.

[23] A. Kibar, H. Karabay, K. Yigit, I. Ucar, H. Erbil, Experimental investigation of inclined liquid water jet flow onto vertically located superhydrophobic surfaces, Experiments in Fluids 49 (5) (2010) 1135-1145.

[24] J. F. Prince, D. Maynes, J. Crockett, Analysis of laminar jet impingement and hydraulic jump on a horizontal surface with slip, Physics of Fluids 24 (10) (2012) 102103-1-15.

[25] J. F. Prince, D. Maynes, J. Crockett, Jet impingement and the hydraulic jump on horizontal surfaces with anisotropic slip,
Physics of Fluids 26 (4) (2014) 042104-1-19.

[26] J. R. Philip, Flows satisfying mixed no-slip and no-shear conditions, Z. Angew. Math. Phys. 23 (1972) 353-372.

[27] J. R. Philip, Integral properties of flows satisfying mixed noslip and no-shear conditions, Z. Angew. Math. Phys. 23 (1972) 960-968.

[28] E. Lauga, H. A. Stone, Effective slip in pressure-driven stokes flow, Journal of Fluid Mechanics 489 (2003) 55-77.

[29] C.-O. Ng, C. Y. Wang, Apparent slip arising from Stokes shear flow over a bidimensional patterned surface, Microfluid Nanofluid 8.

[30] C. Navier, Mémoire sur les lois du mouvement des fluides, Mémoires de l'Acad. R. Sci. Inst. Fr. 6 (1823) 389-440.

[31] R. Hays, J. Crockett, D. Maynes, B. W. Webb, Thermal transport to sessile droplets on superhydrophobic surfaces with rib and cavity features, in: Proceedings of the ASME 2013 International Mechanical Engineering Congress and Exposition, no. IMECE2013-63780, San Diego, California, 2013.

[32] Z. Liu, Y. Gou, J. Wang, S. Cheng, Frost formation on a superhydrophobic surface under natural convection conditions, International Journal of Heat and Mass Transfer 51 (25) (2008) $5975-5982$.

[33] K. K. Varanasi, T. Deng, J. D. Smith, M. Hsu, N. Bhate, Frost formation and ice adhesion on superhydrophobic surfaces, Applied Physics Letters 97 (23) (2010) 234102-1-3.

[34] D. Maynes, J. Crockett, Apparent temperature jump and thermal transport in channels with streamwise rib and cavity featured superhydrophobic walls at constant heat flux, Journal of Heat Transfer 136 (2014) 011701-1-10.

[35] A. Cowley, D. Maynes, J. Crockett, Effective temperature jump length and influence of axial conduction for thermal transport in superhydrophobic channels, International Journal of Heat and Mass Transfer 79 (2014) 573-583.

[36] R. Enright, M. Hodes, T. Salamon, Y. Muzychka, Isoflux Nusselt number and slip length formulae for superhydrophobic microchannels, Journal of Heat Transfer 136 (2014) 012402-1-9.

[37] C.-O. Ng, C. Y. Wang, Temperature jump coefficient for superhydrophobic surfaces, Journal of Heat Transfer 136 (2014) 064501-1-6.

[38] M. Hodes, L. S. Lam, A. Cowley, R. Enright, S. MacLachlan, Effect of evaporation and condensation at menisci on apparent thermal slip, Journal of Heat Transfer 137 (2015) 071502-1-7.

[39] L. S. Lam, C. Melnick, M. Hodes, G. Ziskind, R. Enright, Nusselt numbers for thermally developing Couette flow with hydrodynamic and thermal slip, Journal of Heat Transfer 136 (2014) 051703-1-11.

[40] C. Y. Wang, Benchmark solutions for slip flow and H1 heat transfer in rectangular and equilateral triangular ducts, Journal of Heat Transfer 135 (2013) 021703-1-8.

[41] C. Y. Wang, C.-O. Ng, Natural convection in a vertical slit microchannel with superhydrophobic slip and temperature jump, Journal of Heat Transfer 136 (2014) 034502-1-6.

[42] C.-O. Ng, C. Y. Wang, Natural convection in a vertical microannulus with superhydrophobic slip and temperature jump, Journal of Thermophysics and Heat Transfer 28 (2014) 287-294.

[43] D. Moreira, P. R. Bandaru, Thermal transport in laminar flow over superhydrophobic surfaces, utilizing and effective medium approach, Physics of Fluids 27 (2015) 052001-1-14.

[44] G. Rosengarten, C. Stanley, F. Kwok, Superinsulating heat transfer surfaces for microfluidic channels, in: The Eighteenth International Symposium on Transport Phenomona, 2007.

[45] D. Maynes, B. W. Webb, J. Davies, Thermal transport in a microchannel exhibiting ultrahydrophobic micro-ribs maintained at constant temperature, Journal of Heat Transfer 130 (2008) 022402-1-8.

[46] D. Maynes, B. W. Webb, J. Crockett, V. Solovjov, Analysis of laminar slip-flow thermal transport in microchannels with transverse rib and cavity structured superhydrophobic walls at constant heat flux, Journal of Heat Transfer 135 (2013) 021701$1-10$.

[47] Y. Cheng, J. Xu, Y. Sui, Numerical study on drag reduction 
and heat transfer enhancement in microchannels with superhydrophobic surfaces for electronic cooling, Applied Thermal Engineering 88 (2015) 71-81.

[48] L. S. Lam, M. Hodes, R. Enright, Analysis of Galinstan-based microgap cooling enhancement using structured surfaces, Journal of Heat Transfer 137 (2015) 091003-1-10.

[49] S. V. Patankar, C. H. Liu, E. M. Sparrow, Fully developed flow and heat transfer in ducts having streamwise-periodic variations of cross-sectional area, Journal of Heat Transfer 99 (1977) 180186 .

[50] F. M. White, Viscous Fluid Flow, 3rd Edition, McGraw-Hill, 2006.

[51] F. P. Incropera, D. P. DeWitt, T. L. Bergman, A. S. Lavine, Fundamentals of heat and mass transfer., 6th Edition, John Wiley and Sons, Inc., 2007. 